\title{
ÁREAS Y SUELOS: EL TAMAÑO DE LOS YACIMIENTOS DE SUPERFICIE. UNA PROPUESTA METODOLÓGICA
}

\section{AREAS AND FLOORS: THE SIZE OF SURFACE SITES. A METHODOLOGY PROPOSAL}

\author{
S. PARDO, A. DIEZ Y J. BERNABEU*
}

\begin{abstract}
Resumen: En los estudios sobre el patrón de asentamiento y en los análisis del territorio, la variable "tamaño" del asentamiento juega un papel importante. Considerada como "proxy" de la población, no siempre resulta fácil de calcular en ausencia de estructuras arqueológicas construidas: aterrazamientos o fortificaciones. En este trabajo se presenta una aproximación al cálculo de dicha variable en los asentamientos de superficie caracterizados simplemente por la dispersión de materiales, que toma como punto de partida la correlación entre aquélla y la presencia de los horizontes edáficos ah. La reciprocidad positiva de ambos factores permite sugerir diversas hipótesis sobre la relación entre la formación de tales horizontes y las prácticas agrícolas primitivas.

Palabras claves: Neolítico; Áreas; Marcador edáfico; Valles del Serpis; Microsondeos.
\end{abstract}

\section{CONSIDERACIONES PREVIAS: LA IMPORTANCIA DEL TAMAÑO}

La necesidad de obtener información fidedigna sobre el tamaño de los yacimientos neolíticos al aire libre ${ }^{1}$

\footnotetext{
* Departament de Prehistòria i Arqueología, Universitat de València.

1. Consideramos yacimientos de hábitat al aire libre (poblado o campamento estacional) aquellos espacios que se han habilitado para que el ser humano desarrolle su vida en comunidad. Esta tipología de hábitats pueden presentar diferente morfología, algunos pueden tener cabañas (la Draga; Bosch et al. 2000), otros presentan estructuras excavadas como silos, fosos o cubetas entre otras (Jovades; Pascual 2003), e inclusive ambas. En momentos más avanzados (III milenio) algunos pueden presentar estructuras pétreas (Ereta del Pedregal;
}

Summary: Regarding the studies of settlement pattern and territories in Archaeology, the size of the settlements becomes an important variable. Although it is considered as a proxy of population, it is not always easy to calculate it in absence of archaeological structures: terraces or fortifications. This paper proposes an new method to calculate the size of settlements, when these are characterised simply by the dispersion of material, which takes as its starting point the correlation between the presence of artefacts and soil horizons ah. The positive correlation of both factors suggests several hypotheses about the relationship between the formation of such edaphic horizons and primitive agricultural practices.

Keyworks: Neolithic; Area; Soil Maker; Serpis Basin; Augering.

nos ha sugerido plantear una serie de estrategias para conseguirla. El objetivo principal del presente trabajo lo constituye en primer lugar la elaboración de un sistema para establecer el tamaño de los asentamientos neolíticos al aire libre y en segundo lugar poder establecer la posible relación entre estos y los marcadores edáficos.

\subsection{El "tamaño" como variable}

La variable "tamaño" juega un papel importante en los estudios de arqueología del territorio, sobre todo,

Juan Cabanilles 1994). Finalmente a partir de la Edad del Bronce, generalmente, son lugares amurallados (Mola d'Agres; Peña et al. 1996). 


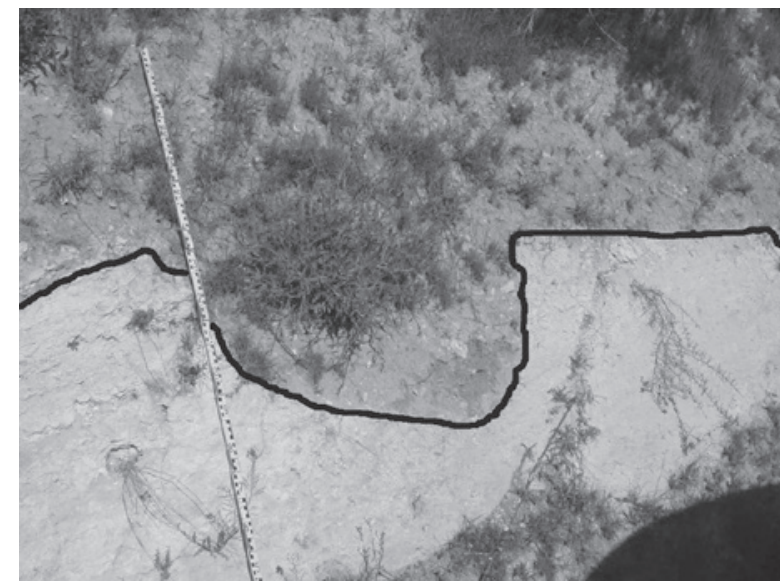

Figura 1. Localización de una estructura negativa (cubeta) en el corte de la carretera. Les Trilles (Cocentaina, Alicante)

para mejorar la comprensión de la organización territorial $^{2}$. Esta variable presenta inconvenientes para su cálculo, principalmente, en los asentamientos de superficie debido no sólo a problemas de índole funcional sino también a complicaciones que derivan básicamente en dos circunstancias.

Las estructuras construidas o estructuras positivas ${ }^{3}$ tienen una duración temporal relativamente corta, sobre todo las cabañas, como se ha comprobado en diferentes trabajos (Coudart 1993; Gómez 2009), exceptuando aquellas estructuras realizadas en piedra (muros y murallas) características de la Edad el Bronce en el País Valenciano.

Las estructuras excavadas ${ }^{4}$ no son reconocibles mediante la prospección superficial, debido a los procesos sedimentarios y los procesos de erosión, pues sólo podemos localizar aquellas observadas en los cortes del terreno (fig. 1).

Estas circunstancias convierten a las estructuras implicadas en "invisibles" a las prospecciones superficiales, debido a que los principales materiales constructivos son perecederos y, por consiguiente, no nos permite interpretar correctamente la organización del espacio. $\mathrm{La}$ acción de factores, bien humanos (p.e. roturaciones) o naturales (p. e. erosión), convierten esta clase de estructuras frágiles a las alteraciones o destrucciones.

Esta "invisibilidad" de las estructuras junto al registro arqueológico neolítico y calcolítico peninsular,

2. Esta variable nos permite observar tanto la jerarquización social del territorio mediante la regla rango-tamaño como las relaciones entre grupos.

3. Entre estas destacamos cabañas, cercados, empaladizas y hogares.

4. Destacamos cubetas, fosos, agujeros de poste, estructuras de combustión y silos entre otras.

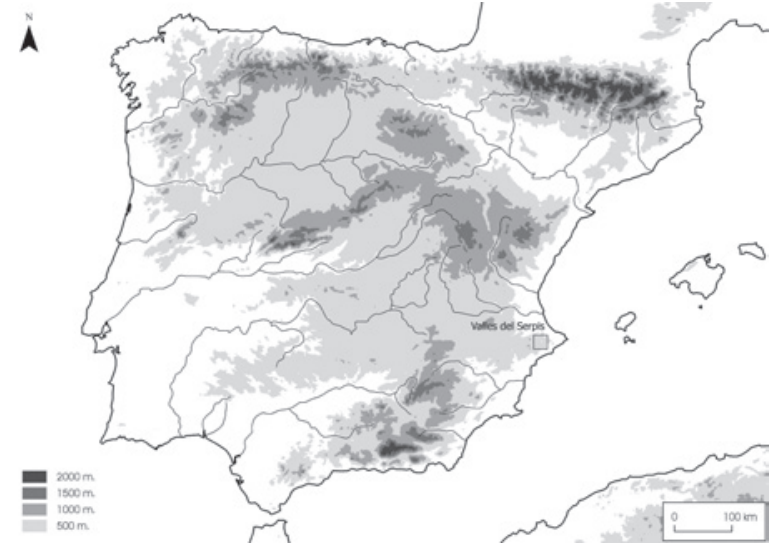

Figura 2. Localización de los valles del Serpis.

caracterizado por la dispersión de material (Bernabeu et al. 1999; Nocete et al. 1995), dificulta el cálculo del tamaño real del yacimiento. La fiabilidad del área es mayor cuando el perímetro del yacimiento esta delimitado mediante la fisiografía como se observa en el yacimiento de la Draga, o bien cuando el sitio está delimitado mediante un recinto amurallado, como ocurre en la Mola d'Agres. La dispersión de material, como se ha observado en un trabajo reciente (Mata et al. e.p) proyecta unos resultados menos fiables debido a problemas tanto deposicionales como postdeposicionales. Tal vez estos problemas han influido decisivamente a la hora de plantear y realizar trabajos para establecer la extensión de los yacimientos al aire libre. Los hábitats neolíticos donde se ha realizado una aproximación del área mediante excavación arqueológica son pocos, así destacamos en los valles del Serpis los yacimientos de Niuet (Bernabeu et al. 1994) y Jovades (Bernabeu 1993; Pascual 2003) entre otros, y también yacimientos de valles vecinos como el de Colata en la Vall d'Albaida (Gómez et al. 2004) o el Arenal de la Virgen (Fernández López et al. 2008). No obstante, el problema del área puede ser minimizado mediante la asociación de las dispersiones superficiales con otros indicadores como los marcadores edáficos. Esta asociación permite obtener una mayor fiabilidad del tamaño aproximado de los asentamientos analizados.

Nuestro trabajo sistemático en los valles del Serpis (Bernabeu et al. 2008) (fig. 2) nos revela la posible existencia de una relación directa entre los horizontes húmicos ah subsuperficiales y los lugares de hábitat, como veremos más adelante. En las excavaciones del Mas d'Is (Bernabeu et al. 2003) y Regadiuet (García Puchol et al. 2008) la presencia de horizontes edáficos 


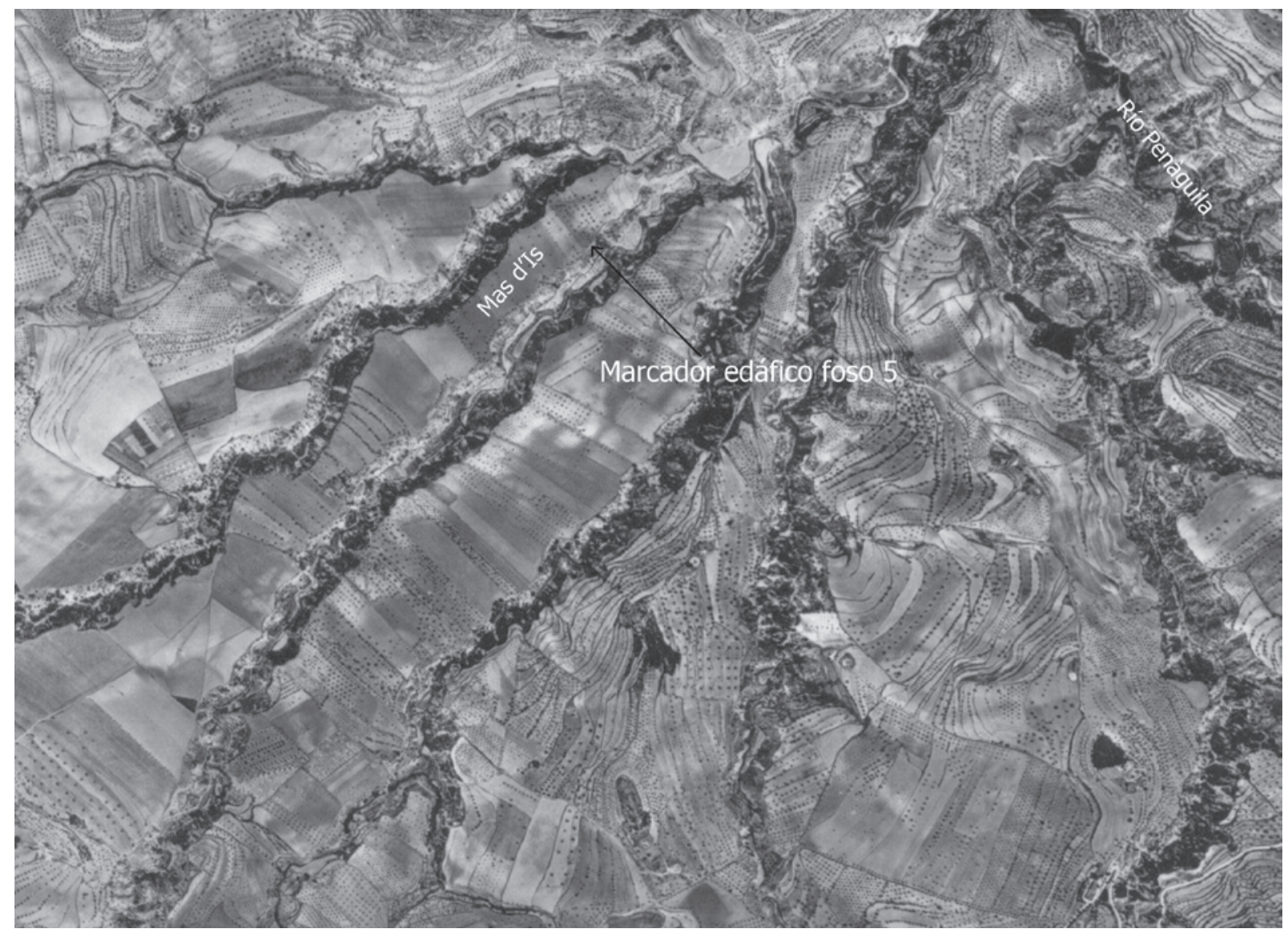

Lámina I. Fotografía aérea del paraje de "les Puntes" donde se observan los elementos pedográficos del Mas d'Is (Penáguila, Alicante).

ah parece un factor decisivo para el establecimiento de sus primeros pobladores, al mismo tiempo que la actividad humana originó un continuado aporte de materia orgánica, incrementando las diferencias de coloración entre las zonas ocupadas y las colindantes. Por otra parte, los fosos monumentales del Mas d'Is se observan claramente en las fotografías aéreas debido a un doble factor: por un lado el proceso de relleno natural una vez abandonados y, por otro, tanto la aportación antrópica como la circulación subsuperficial ha generado un enriquecimiento considerable de la materia orgánica (lám. I).

\section{CÁLCULAR EL ÁREA: PROPUESTA METODOLÓGICA}

Debido al gran número de yacimientos neolíticos documentados en los diferentes trabajos de prospección arqueológica en los valles del Serpis (Bernabeu et al. 2008) nos hemos planteado elaborar un sistema para calcular el área de aquellos reconocidos sólo por este medio, la prospección superficial.

\subsection{Una primera aproximación: la solución geométrica}

El primer intento de cálculo del área de los yacimientos, a partir de la dispersión de material arqueológico, está en consonancia con el desarrollo de los trabajos de campo, ya que junto a los parámetros descriptivos básicos (localización, coordenadas, hallazgos y estructuras) de la ficha de campo, nos planteamos incorporar otro parámetro relacionado con las dimensiones del asentamiento para incorporar nueva información a la ficha utilizada en los trabajos de prospección. Así que esbozamos una solución geométrica, esta se puede realizar mediante el Convex Hull o el Concave Hull.

ISSN: 1133-4525 ISSN-e: 2255-3924 


\subsubsection{El Convex Hull}

La delimitación del área del yacimiento se calculó a partir de la expresión mínima del registro de la colección arqueológica documentada mediante la prospección superficial, es decir, mediante lo que la bibliografía arqueológica especializada llama convex hull o envolvente mínima convexa (Min y Zheng 1993). Este método realiza un polígono convexo con los puntos más exteriores de la agrupación de material arqueológico analizada, crea una determinada forma donde cualquier artefacto arqueológico documentado en la prospección queda dentro del área realizada. Este procedimiento, el convex hull, se puede realizar manualmente, aunque es muy costoso, o de manera automatizada con la utilización de cualquier Sistema de Información Geográfica $\mathrm{SIG}^{5}$ con el algoritmo disponible.

Una vez realizada esta solución nos planteamos si esta fórmula para calcular el área de los yacimientos está afectada por las condiciones de visibilidad. En primer lugar, existe numerosa literatura al respecto (Renfrew y Bahn 1993; García Sanjuán 2005), coincidiendo en la importancia de la visibilidad y las condiciones meteorológicas en el resultado final de la muestra recogida. No obstante, en los proyectos de prospección sistemáticas que nos afectan, esta cuestión no es tan determinante, ya que los yacimientos documentados son visitados en reiteradas ocasiones. Así que la muestra total resultante la consideramos válida para poder establecer el área de los yacimientos.

\subsubsection{El Concave Hull}

La delimitación del área del yacimiento se calcula a partir de la expresión mínima de registro del material arqueológico localizado en la prospección superficial, es decir, mediante lo que la bibliografía especializada llama concave hull o envolvente mínima cóncava (Moreira y Santos 2007). Este método consiste en unir mediante un polígono cóncavo los puntos más exteriores de la agrupación de elementos arqueológicos que se quiere analizar, unión que forma una determinada área donde cualquier elemento arqueológico documentado en la prospección queda dentro de la extensión realizada. Este procedimiento de establecer el área de los yacimientos se puede realizar de forma manual, aunque es muy costoso, si bien también cabe la posibilidad

5. GVSIG 1.9 con la adicción de Sextante. Software open source y desarrollado por la Generalitat Valenciana. www.gvsig.gva.es de efectuarla de manera automatizada con la utilización de cualquier programa informático ${ }^{6}$ con este algoritmo disponible. Este método de cálculo del área no se ha utilizado en arqueología.

\subsection{La delimitación mediante los marcadores edáficos}

Los marcadores edáficos o indicadores pedológi$\cos$ son alteraciones que se reflejan preferentemente en la diferenciación del color. Esta coloración disímil está relacionada con factores antrópicos o naturales (Porta 2003). Si bien los primeros se asocian con el aporte de materia orgánica producido por las actividades realizadas reiteradamente en determinados lugares, los segundo presentan causas edafogénicas, como son las propias características de los suelos, destacando en los valles del Serpis la formación cíclica a lo largo del Holoceno de horizontes húmicos $\mathrm{ah}^{7}$ que ponen de manifiesto la existencia de cantidades de materia orgánica. La conservación de ambos tipos tendría una explicación geomorfológica. Por otra parte, destacamos que la coloración observada en la fotointerpretación puede estar relacionada con el grado de humedad y la circulación subsuperficial del agua, ya que los rellenos sedimentarios de los paleocauces son comparativamente poco compactos.

En las numerosas ocasiones que visitamos los yacimientos documentados en los valles del Serpis observamos que, generalmente, el material arqueológico se localizaba dentro de determinadas manchas del suelo, manchas observadas tanto en el mismo trabajo de campo como en las fotografías aéreas, así que nos planteamos realizar otro enfoque en la delimitación de los yacimientos. Este método de delimitación fundamenta su argumentación en la consideración del marcador edáfico como la extensión máxima del yacimiento.

El método consiste en establecer el área de los asentamientos superficiales mediante los marcadores edáficos, pues estos nos pueden ayudar a distinguir si una

6. ConcaveHull versión 0.9.1. disponible en http://concavehull.com

7. La formación de estos suelos (A) está en relación con la acumulación de materia prima, sin embargo, la materia orgánica esta presente en forma de compuestos muy evolucionados formando humus íntimamente ligado a la fracción mineral. Los suelos AH son suelos con una importante base de materia orgánica en horizontes minerales. Estos suelos se reconocen por la presencia de manchas oscuras que contrastan agudamente con los colores claros relacionados con la presencia de margas in situ o redepositadas. Por otro lado estos suelos AH también son distinguibles de los suelos arcillosos y rojos del horizonte B. 
Figura 3. Representación del área del yacimiento ac-301 (Gorga, Alicante) mediante la utilización de los diferentes métodos explicados en el texto.

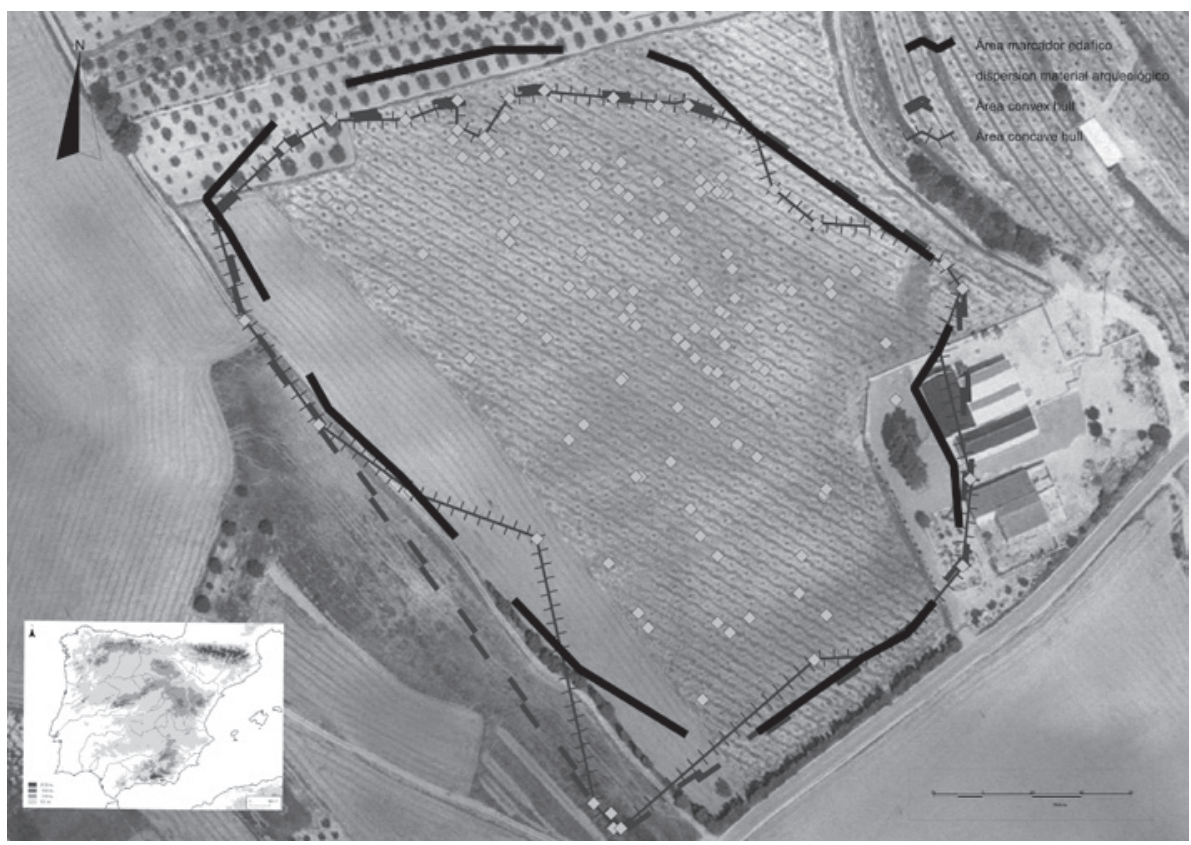

determinada área ha sido utilizada reiteradamente en el pasado. En la siguiente figura se observa el área mediante la superposición de los diferentes métodos para calcular el tamaño (fig. 3).

\subsubsection{Comprobando la hipótesis: los microsondeos}

Expuesta la relación entre asentamiento y marcador edáfico planteamos si toda la mancha edáfica tiene material arqueológico, así que realizamos un trabajo experimental durante el mes de agosto de 2007 en el yacimiento del Mas del Pla (lám. II) para dar respuesta a la pregunta planteada, siendo ésta la primera intervención arqueológica en el yacimiento desde su identificación como lugar arqueológico en los años 80 del siglo pasado dentro del programa de prospecciones sistemáticas en los valles del Serpis. El yacimiento se localiza en el término municipal de Penàguila (Alicante) $\mathrm{y}$, más concretamente en el paraje conocido como les Puntes de Benifallim. Los materiales recuperados tanto en las diferentes visitas al yacimiento como en nuestra intervención arqueológica le atribuyen no sólo una cronología relacionada con el Neolítico inicial (NIB) o avanzados (ІІB), sino también con etapas protohistóricas (Cultura Ibérica).

A partir del patrón de distribución de los materiales documentados en la prospección se realizaron una serie de microsondeos en columna de tramos de $10 \mathrm{~cm}$ (al igual que en el yacimiento de Regadiuet [García Puchol et al. 2008]) que resultaron determinantes a la hora de establecer nuestra hipótesis de trabajo. En el Mas del Pla se practicaron un total de 31 augers o microsondeos colocados de forma radial (fig. 4), posibilitando rastrear todo el marcador edáfico y su zona colindante para documentar la existencia de material arqueológico, al mismo tiempo que nos permitía documentar el depósito arqueológico del yacimiento. La profundidad alcanzada por los diferentes microsondeos fue variada, ya que estuvo condicionada por la presencia de piedras o bien el depósito natural.

$\mathrm{Al}$ exterior del marcador edáfico se realizaron un total de 14 microsondeos, éstos aclararon nuestra hipótesis de trabajo ya que no se documentó material arqueológico. En la zona interior del marcador edáfico se realizó un total de 17 microsondeos con desigual resultados.

- La profundidad donde se ha documentado el material arqueológico es variable.

- No todos los microsondeos realizados en el interior del marcador edáfico presentan material arqueológico.

El primer problema se refiere a la existencia de dos tipos de microsondeos con material arqueológico, un primer grupo donde el material se encuentra en tramos superiores y un segundo grupo que lo presenta en tramos inferiores. ¿La distribución del material arqueológico

ISSN: 1133-4525 ISSN-e: 2255-3924 


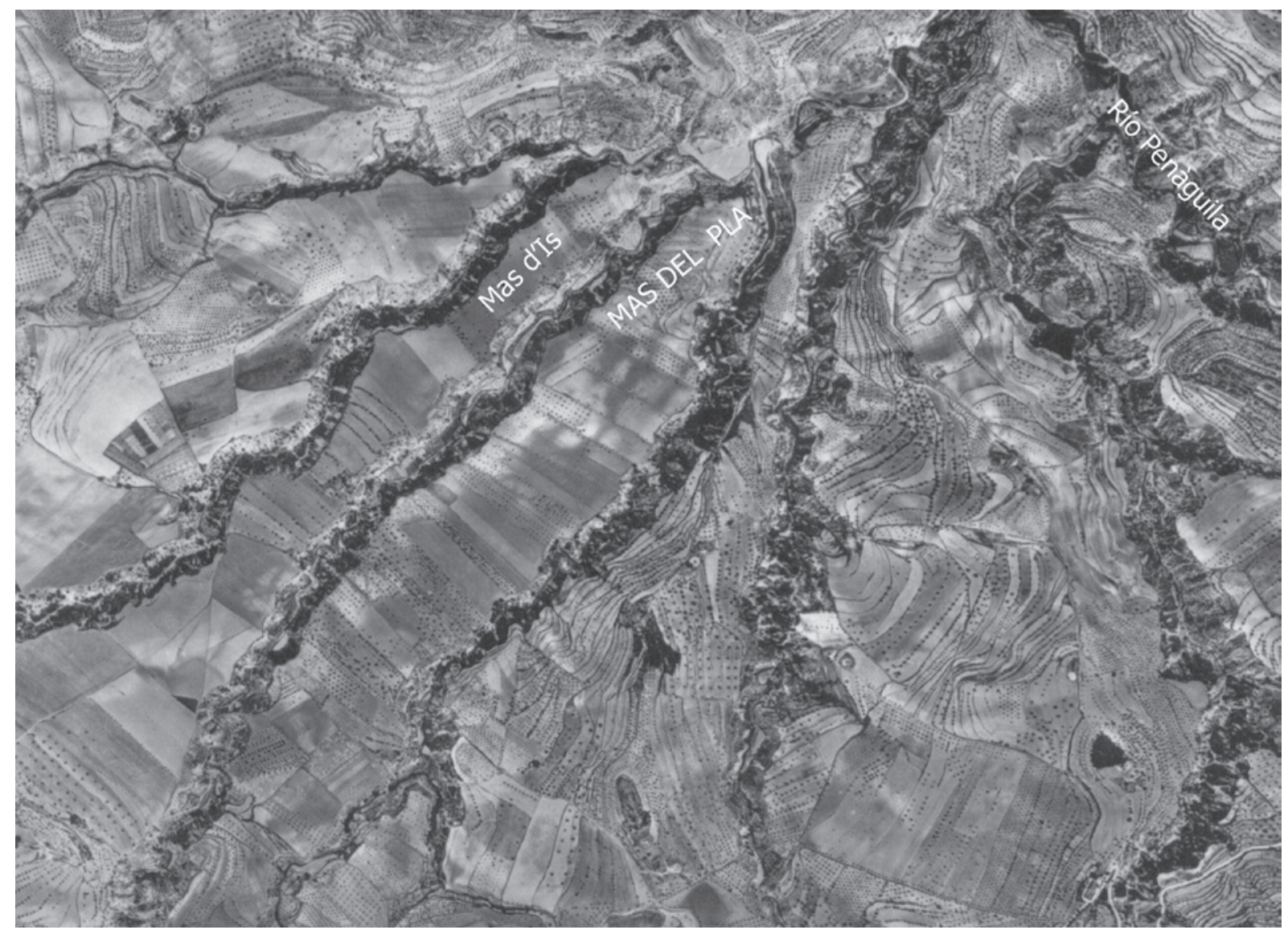

Lámina II. Localización del yacimiento del Mas del Pla (Penáguila, Alicante).

ha sido alterada por los procesos postdeposicionales naturales o antrópicos? Destacamos entre los primeros la erosión, que actúa a gran escala, pudiendo alterar la distribución del material arqueológico. En los valles del Serpis, la naturaleza margosa del sustrato ha facilitado el movimiento del material arqueológico, aunque éste nunca recorre grandes distancias respecto a las posiciones originales, como ratifica la escasez de material rodado en las colecciones superficiales. Entre los factores antrópicos señalamos el aterrazamiento, que actúa a una escala similar a los factores naturales, es decir, existe un movimiento de material desde las posiciones originales, pero este material nunca recorre gran distancia a la vez que, con el paso del tiempo, este movimiento decrece considerablemente (Cowan y Odell 1990). Nuestro enfoque para solucionar este primer problema, teniendo en cuenta los procesos postdeposicionales, ha sido descartar aquellos microsondeos que tienen sólo materiales arqueológicos en los tamos superiores (tramo $1 \mathrm{y}$ tramo 2), es decir, aquellos donde el material arqueológico se localiza en los 20 primeros centímetros de la superficie. Sin embargo, si el auger tiene restos arqueológicos en los tramos superiores e inferiores éste es considerado para la elaboración del área, pues aceptamos que los tramos inferiores de los microsondeos no han sufrido alteraciones, aunque siempre existen problemas de bioturbaciones.

El segundo problema concierne a dos tipos de microsondeos, un primer grupo donde los augers, situados dentro del marcador edáfico, son estériles, es decir, que no presentan material arqueológico, y un segundo grupo que si lo presentan. La solución, teniendo en cuenta el primer problema, ha sido delimitar un área con los microsondeos más exteriores con material arqueológico mediante el convex hull (fig. 5).

Los resultados obtenidos nos plantean que a priori no se puede determinar el área atendiendo sólo a los marcadores edáficos, ya que el área obtenida mediante los microsondeos corresponde sólo a una parte del marcador. La solución ha sido realizar un sistema de ponderación del área, de manera que al yacimiento del Mas del Pla se le pueda atribuir un área a partir de nuestra 


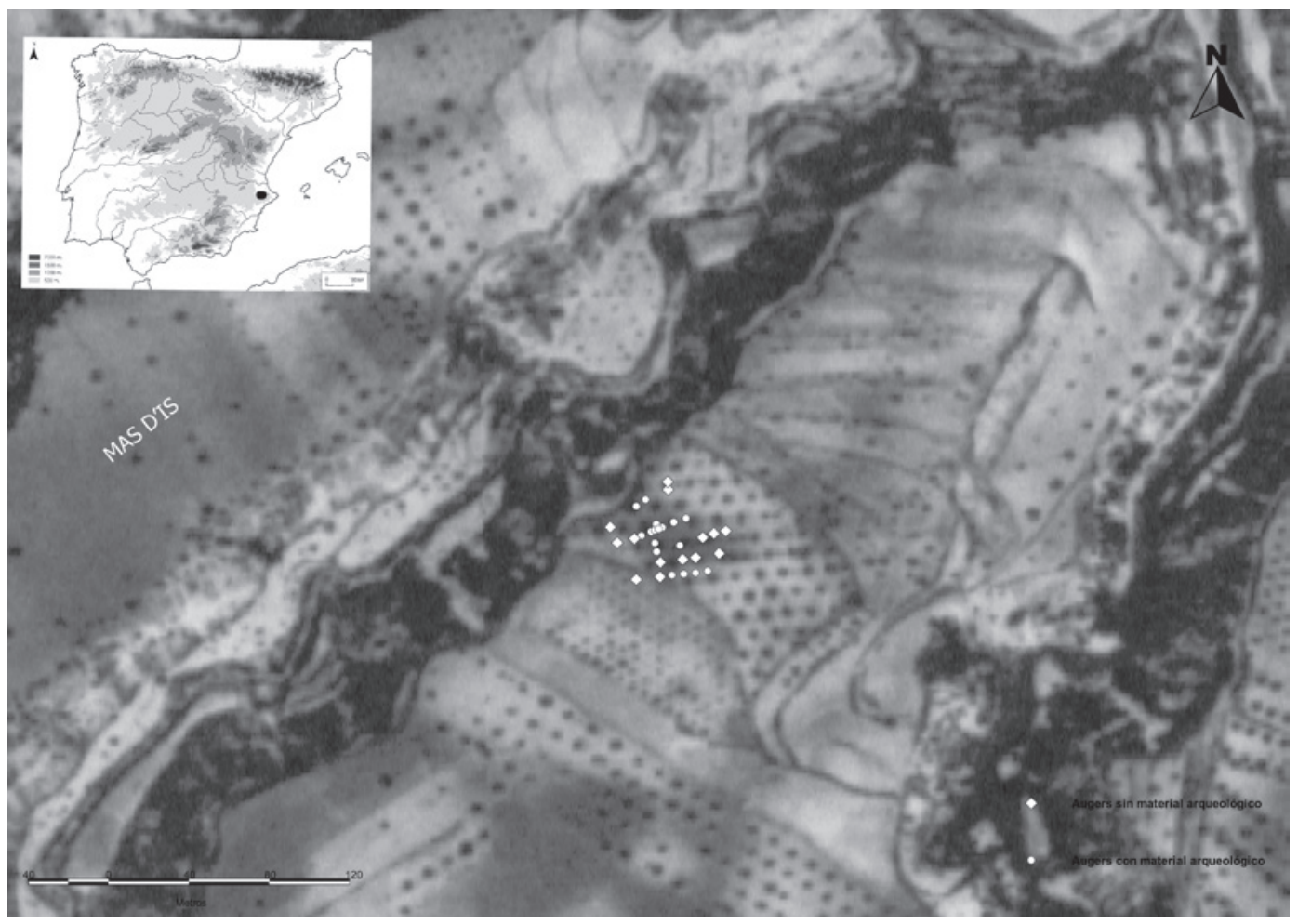

Figura 4. Localización de los microsondeos realizados en el Mas del Pla (Penáguila, Alicante).

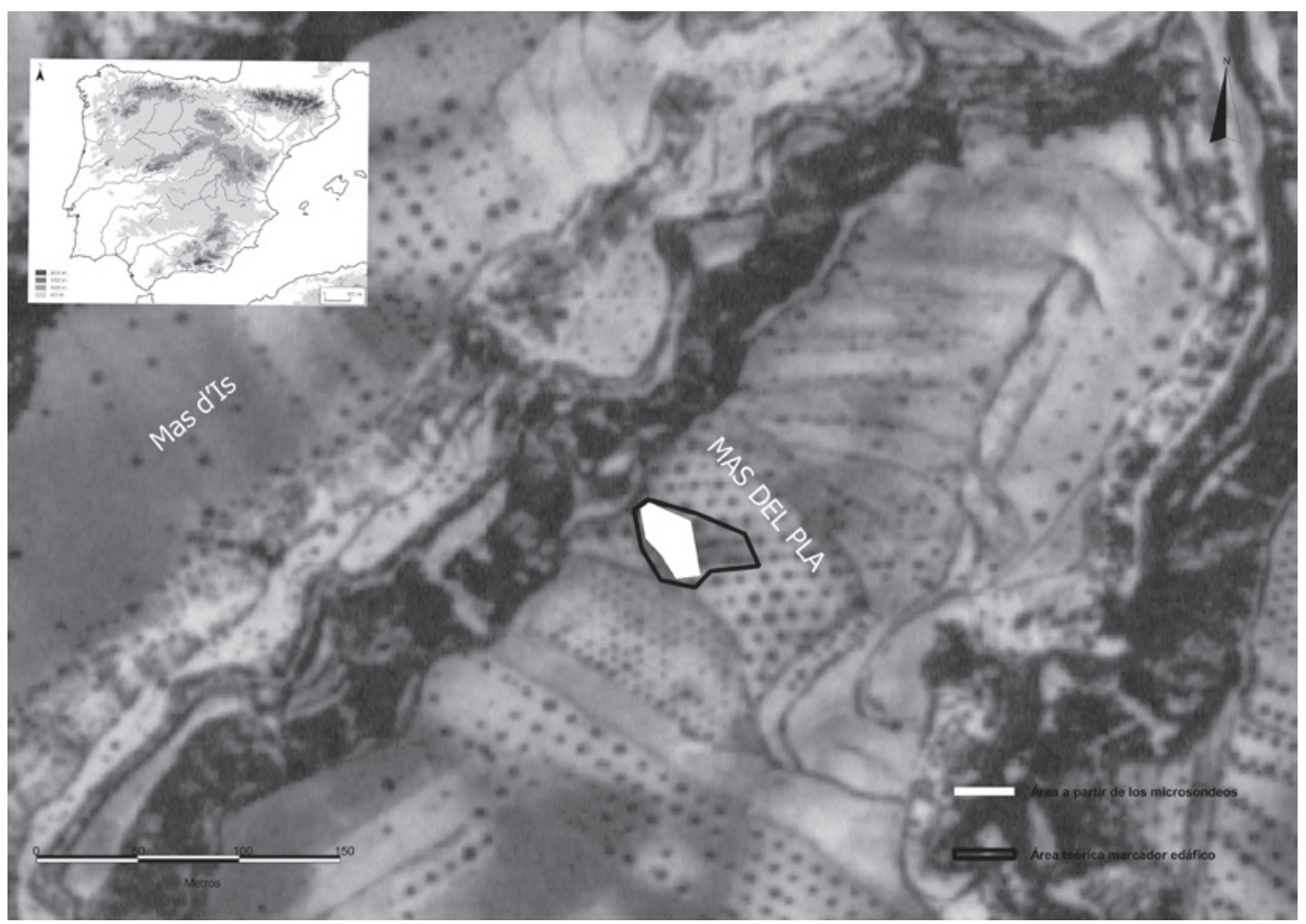

Figura 5. Cálculo del área del Mas del Pla (Penáguila, Alicante) a partir de los resultados de los microsondeos. En negro área calculada mediante marcador edáfico, en blanco área a partir de macrosondeos. 
intervención. Este sistema consiste, en primer lugar, en fijar el marcador edáfico como el Área Total Ocupada (a partir de ahora ATO) y, por otra parte, calcular el Área de los Microsondeos con Material Arqueológico (a partir de ahora AMMA). La ponderación consiste en establecer la relación entre el ATO y AMMA, siendo la fórmula resultante $\left(r^{\prime}\right)=$ AMMA / ATO, que muestra la proporción del tamaño del ATO y el AMMA.

\section{CORRELACIÓN Y TENDENCIAS DE OCUPACIÓN DE LOS SUELOS}

En la descripción de la selección del suelo para establecer el lugar de habitación utilizaremos variables cualitativas.

\subsection{Tablas de contingencia y asociación de los resultados}

La representación de los casos analizados en este trabajo es mediante las tablas de frecuencias o tablas de contingencia. Este tipo de tablas nos permite obtener una información más precisa de la existencia de una asociación entre las variables, o si por el contrario se pueden considerar independientes. Es decir, nos permite analizar la proporción de casos para cada categoría de una de las variables es independiente del valor que toma la otra variable. En nuestro caso (tabla 1), deseamos conocer si la proporción de yacimientos documentados en cada una de las categorías de suelo es diferente según el momento cronológico analizado o si, por el contrario, se pueden considerar independientes.

Tabla 1.

Yacimientos documentados en cada momento cronológico y la división de los yacimientos utilizando el criterio de presencia o ausencia de horizonte húmico Ah.

\begin{tabular}{|c|c|c|c|}
\hline Cronologí & $\begin{array}{c}\text { Total } \\
\text { YACIMIENTOS }\end{array}$ & $\begin{array}{c}\text { Presencia } \\
\text { HORIzonte ah }\end{array}$ & $\begin{array}{c}\text { Ausencia } \\
\text { HORIzonTE ah }\end{array}$ \\
\hline NIA & 8 & 7 & 1 \\
\hline NIB & 17 & 15 & 2 \\
\hline NIC & 14 & 14 & 0 \\
\hline NIIA & 5 & 4 & 1 \\
\hline NIIB & 34 & 29 & 5 \\
\hline$H C T$ & 9 & 9 & 0 \\
\hline$B Z$ & 35 & 15 & 20 \\
\hline
\end{tabular}

El cálculo de la v de $\mathrm{Cramer}^{8}$ nos permite observar si la asociación entre las variables es significativa, o por el contrario no existe ninguna correlación. Los resultados obtenidos nos proporcionan una información interesante para describir las tendencias en la selección de los suelos por parte de las primeras comunidades agrícolas.

\subsection{Interpretación de las tendencias en la ocupación del territorio}

La gráfica (fig. 6) resume la información relativa a la selección de los suelos en los valles del Serpis durante la Prehistoria Reciente. La representación muestra la relación entre los yacimientos documentados y la selección del suelo. Los resultados se presentan por horizontes cronológicos. Éste gráfico presenta dos categorías, la primera ( $\mathrm{SAH}$ ) corresponde a los yacimientos donde existe una relación entre dispersión de material y marcador edáfico, la segunda categoría (NAH) hace referencia a los yacimientos situados en suelos sin horizontes húmicos ah. La gráfica nos muestra la existencia de una relación directa entre los asentamientos neolíticos al aire libre y los suelos con presencia de marcadores edáficos, correlación que desaparece con la llegada de la Edad del Bronce.

Durante el Neolítico se observa la existencia de una selección de los suelos para establecer las aldeas agrícolas. Analizando la gráfica observamos claramente la selección de los suelos con presencia de ah. Es razonable interpretar esta elección como consecuencia del nuevo sistema de gestión del territorio basado en aldeas agrícolas situadas en las zonas de cultivo. Esta selección de los suelos esta ligada a dos aspectos fundamentales como el mejor rendimiento agrícola y su facilidad a la hora de labrarlos. El cambio en la elección de los suelos se documenta en la Edad del Bronce. El incremento de la categoría (NAH) es superior respecto a la dinámica documentada a lo largo del Neolítico, como puede verse en la figura 6. Este incremento sugiere la consolidación del poblamiento en las zonas altas de los valles, aunque, el modelo de asentamientos junto a la zonas de cultivo, característico del Neolítico, persiste ${ }^{9}$ de manera secundaria.

8. PAST versión 2.0 disponible en http://folk.uio.no/ohammer/ past/download.html

9. Aunque aún no se conocen muchos ejemplos de este tipo de yacimientos, tanto las excavaciones del Mas del Sanç, Albocasser (Fernández et al. 2004) como Torre de la Sal, Oropesa (Flors 2009) han aportado evidencias, auque aisladas, de un poblamiento en llano. 
Figura 6. Gráfica donde se observa la relación entre los yacimientos documentados sobre horizonte húmico $\mathrm{Ah}$ y aquellos que no lo están.

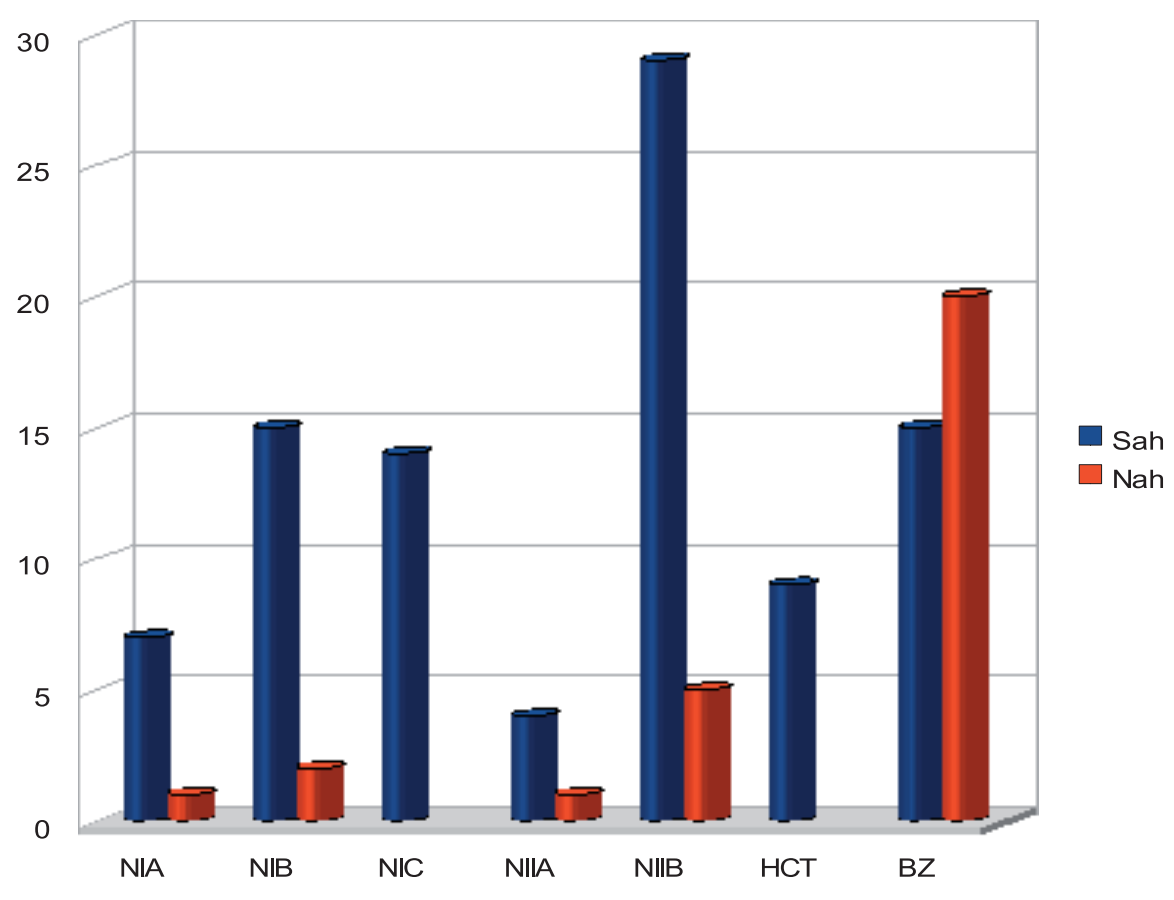

\section{DISCUSIÓN}

Los diferentes trabajos realizados en los valles del Serpis ponen en manifiesto distintas cuestiones que atañen tanto a problemas de interpretación de la dispersión del material arqueológico en las prospecciones como la relación de los lugares de hábitat al aire libre y los suelos con horizontes húmicos ah. El presente trabajo ha intentado establecer una propuesta metodológica para poder resolver los problemas planteados.

La delimitación del área mediante los marcadores edáficos en los valles del Serpis es un sistema válido a partir de los resultados obtenidos en el Mas del Pla, no obstante aceptamos las restricciones empíricas existentes en nuestro trabajo de campo, ya que sólo se ha realizado microsondeos destinados al estudio de la delimitación del área en este yacimiento. Debido a los pocos ejemplos en el estudio del tamaño podemos considerar este trabajo como un ensayo preliminar para solucionar la problemática del área de los yacimientos mediante las prospecciones. Sin embargo debemos afianzar esta hipótesis mediante la incorporación de los resultados de los yacimientos excavados y realizar otros estudios enfocados ${ }^{10}$ al cálculo del tamaño. A falta de

10. . Entre los yacimientos excavados se puede realizar diferentes análisis para observar la evolución del área, así por ejemplo, estos estudios que nos permitiría perfeccionar el método de cálculo planteamos la posibilidad de extrapo$\operatorname{lar}^{11}$ los resultados obtenidos en el Mas del Pla a los asentamientos neolíticos de superficie documentados en los valles del Serpis.

En relación a la formación de los suelos con horizontes húmicos ah, las diferentes excavaciones de yacimientos de cronología neolítica en los valles del Serpis plantean la siguiente cuestión ¿Los suelos con horizontes ah están formados cuando se establecen las primeras sociedades agrícolas? Actualmente existen dos grandes líneas

podemos efectuar un estudio de los silos documentados en cualquier poblado de silos, característico del III milenio, para observar cuantas estructuras funcionan durante el mismo horizonte cronológico y comprobar cual es la extensión máxima del yacimiento.

11. La extrapolación consiste en establecer la relación entre el ATO y la ponderación del área del Mas del Pla al multiplicar el ATO por dicha ponderación. La fórmula resultante es $\left(w^{\prime}\right)=$ ATO x 0'5762, que muestra la proporción del ATO y la ponderación del Mas del Pla. Ésta extrapolación nos ayudará a realizar una aproximación sobre la extensión ocupada por los poblados al aire libre a lo largo de todo el Neolítico en los valles del Serpis. Actualmente, si exceptuamos el yacimiento de Benàmer, aún inédito, no existen yacimientos excavados en su totalidad para observar si existen divergencias entre el área conocida mediante la excavación y el tamaño calculado mediante este método, la asociación marcador edáfico/dispersión de material. Sin embargo esta extrapolación nos permite, hoy por hoy, utilizar los resultados para iniciar nuevos análisis territoriales en los valles del Serpis utilizando entre otros variables, el "tamaño". 
para explicar la formación de los horizontes húmicos ah respecto a las primeras sociedades agricultoras.

Hipótesis antropogénica: Los horizontes húmicos ah no estarían formados cuando se establecen las primeras sociedades neolíticas. Estas sociedades producirían una cantidad elevada de materia orgánica proveniente, en primer lugar, del aporte de los carbones al quemar la vegetación, pero también de todo tipo de desechos asociados a las actividades realizadas en el entorno de las viviendas, así como durante el proceso de amortización de éstas (Stevanovic 1997; Wilson et al. 2009). Procesos similares de formación de los suelos se han documentado tanto en la selva amazónica, en los llamados Terra Preta Soils ${ }^{12}$ (Lehmann et al. 1993) como en zonas del mediterráneo europeo (Nicosia et al. e.p.). Este supuesto antropogénico explicaría la documentación en yacimientos como Mas d'Is de estructuras de combustión en las bases de estos horizontes húmicos ah.

Otra visión sobre la formación de los horizontes húmicos ah como consecuencia de la actividad humana es la que entrevé dicha formación como un proceso producido una vez abandonado el lugar de hábitat. Esto nos pone ante un panorama en que esos lugares fueron dejados en baldío por unos centenares de años ${ }^{13}$ (tiempo necesario para el desarrollo del horizonte edáfico ah), Esta formación de los suelos explicaría la razón por la que existe diferente coloración dentro de los mismos marcadores edáficos; obviamente las estructuras excavadas (por ejemplo silos y fosos) serían colmatadas, una vez abandonadas, convirtiéndose en zonas deprimidas que funcionarían como trampas de agua (debido al mal drenaje de las margas) y sedimentos en los que se desarrollarían horizontes húmicos $\mathrm{ah}^{14}$. Sin embargo, también cabe que lo interpretado por los edafólogos como tal sea algo que simplemente se le parece tratándose en realidad de una signatura de la ocupación humana.

12. Somos concientes en todo momento que el aporte de carbones y las condiciones edafológicas son muy diferentes en la selva amazónica y los valles del Serpis, debido al grado de vegetación, siendo esta la razón por la que los horizontes amazónicos son más negros que los horizontes del Serpis. Sin embargo, las muestras de sedimento recuperados en las excavaciones del Mas d'Is como el Regadiuet dan positivo en presencia de carbones. Por otra parte, esta hipótesis no implica la aceptación de la ignicultura por parte de los primeros agricultores.

13. Una vez abandonado en lugar de hábitat, junto a factores climáticos existe una desintegración de la materia orgánica. Esta descomposición aporta al suelo diferentes minerales y gases que son esenciales para el desarrollo del horizonte $\mathrm{AH}$. El tiempo necesario para la formación de estos suelos oscila entre 1-1000 años (Porta 2003).

14. Esta acumulación de sedimentos junto a la propia actividad biológica crea el ambiente oportuno para la formación de estos suelos.
Hipótesis de ruptura: Los horizontes húmicos ah de los suelos estarían en desarrollo en el momento de la llegada de los primeros agricultores. Estas primeras sociedades agropecuarias ocuparían los horizontes húmicos ah por razones de fertilidad, pero, tanto la erosión en los inicios del Neolítico (Fumanal 1990) como los reiterados trabajos relacionados con la labranza de los campos, supusieron una ruptura en la formación de los horizontes húmicos ah, desarrollo que continuará una vez abandonados los lugares de hábitat.

Somos concientes que las disquisiciones desarrolladas en las líneas anteriores son una aproximación para poder contestar a una pregunta inicial compleja. También cabe la posibilidad que los dos escenarios expuestos hayan podido ocurrir de manera combinada. Sin embargo, planteamos la necesidad de realizar estudios multidisciplinares en esta clase de suelos (donde se ha documentado presencia humana) mediante la realización de estudios micro-sedimentológicos utilizando técnicas propias de la química puedan desbloquear el complicado debate en torno a la formación de estos suelos.

\section{BIBLIOGRAFÍA}

BERNABEU AUBÁN, J. (1989): La tradición cultural de las cerámicas impresas en la zona oriental de la Península Ibérica, Trabajos varios del SIP 86. València.

- (dir.) (1993): El III milenio aC en el País Valenciano. Los poblados de Jovades (Cocentaina) y Arenal de la Costa (Ontinyent), Ajuntament d'Ontinyent, Ajuntament de Cocentaina i Universitat de València.

BERNABEU, J.; DÍEZ, A.; OROZCO, T.; GÓMEZ, M. y MOLINA, J. (2003): "Mas d'Is (Penàguila, Alacant): Aldeas y recintos monumentales del neolítico inicial en el valle del Serpis", Trabajos de Prehistoria 60 (2): 39-59.

BERNABEU, J.; GARCÍA, O.; LA ROCA, N. y BARTON, M. (1999): "Prospecciones sistemáticas en el valle del Alcoi (Alicante): primeros resultados", Arqueología Espacial 21: 29-64.

BERNABEU, J y MOLINA, LL. (ed.) (2009): La cova de les Cendres (Moraira-Teulada, Alicante), Marq Serie Mayor 6.

BERNABEU, J.; MOLINA, LL.; DÍEZ, A.; OROZCO, T. y BARTON, C.M. (2008): "Los valles del Serpis (Alicante): 20 años de trabajo de campo", en M.S. Hernández, J.S. Soler y J.A. López (eds.), 
IV congreso del Neolítico Peninsular (Alicante, 2006). Museo Arqueológico de Alicante (1): 50-57.

BERNABEU, J.; PASCUAL, J.LL.; OROZCO, T.; BADAL, E.; FUMANAL, M.P y GARCÍA, O. (1994): "Niuet (L'Alqueria d'Asnar). Poblado del III milenio a.C", Recerques del Museu d'Alcoi 3: 9-74.

BOSCH, A.; CHILLIDA, J. y TARRÚS, J. (2000): El poblat lacustre neolític de la Draga. Excavacions de 1990 a 1998, Monografies Casc 2.

COUDART, A. (1993): "De l'usage de l'architecture domestique et de l'anthropologie sociale dans l'approche des sociétés néolithique: l'exemple du Néolithique danubien", en Le Néolithique du nordest de la France et des regions limitrophes. Actes du XIIIé colloque interrégional sur le Néolithique (Metz 10-12 octobre 1986): 114-135.

COWEN, F. y ODELL, G.H. (1990): "More o Estimating tillage efectts: Reply to Dunnell and Yorston", American Antiquity 55: 598-605.

FERNÁNDEZ LÓPEZ, J.; GÓMEZ, M.; DÍEZ, A.; FERRER, C. y MARTÍNEZ, A. (2008): "Resultados preliminares del proyecto de investigación sobre los orígenes del neolítico en el Alto Vinalopó y su comarca: la revisión de el Arenal de la Virgen (Villena, Alicante)", en M.S. Hernández, J.S. Soler y J.A. López (eds.), IV Congreso del Neolítico Peninsular (Alicante, 2006). Museo Arqueológico de Alicante (1): 107-116.

FERANDEZ, J.; GARCÍA, R.; GUILABERT, A.P.; GUILLEM, P.M; MOLINA, LL. y PÉREZ, R. (2004): "Mas de Sanç, un nuevo yacimiento a aire libre con estructuras de almacenamiento de la Edad del Bronce en el Parc Cultural Valltorta Gasulla (Castellón), en M.S. Hernández y L. Hernández (ed.), La Edad del Bronce en Tierras Valencianas y Zonas limitrofes. Ayuntamiento de Villena. Instituto alicantino de Cultura Juan GilAlbert. Villena.

FLORS, E. (coord.) (2009): Torre la Sal (Ribera de Cabanes, Castellón). Evolución del paisaje antrópico desde la prehistoria hasta el Medievo. Monografies de Prehistòria i Arqueologia Castellonenques 8.

FUMANAL, M.P. (1990): "Dinámica sedimentaria holocena en valles de cabecera del País Valenciano", Cuaternario y Geomorfología 4: 93-106.

GARCÍA, O.; BARTON, C.M. y BERNABEU, J. (2008): "Programa de prospección geofísica, microsondeos y catas para la caracterización de un gran foso del IV milenio cal. AC en el Alt del Punxó (Muro de l'Alcoi, Alacant)", Trabajos de Prehistoria 65 (1): 143-154.
GARCÍA, O.; DÍEZ, A.; BERNABEU, J. y LA ROCA, N. (2008): "El yacimiento prehistórico del Regadiuet: datos preliminares de la secuencia meso-neolítica", en M.S. Hernández, J.S. Soler y J.A. López (eds.), en $I V$ congreso del Neolítico Peninsular (Alicante, 2006). Museo Arqueológico de Alicante (1): 70-78.

GARCÍA SANJUÁN, L. (2005): Introducción al reconocimiento y análisis arqueológico del territorio. Ariel Prehistórica.

GÓMEZ, M.; DÍEZ, A.; VERDASCO, C.; GARCÍA PUCHOL, O.; OROZCO, T.; PASCUAL, J.LL.; CARRIÓN, Y. y PÉREZ, G. (2004): "El yacimiento de Colata (Montaverner, Valencia) y los poblados de silos del IV milenio en las comarcas centro-meridionales del País Valenciano", Recerques del Museu d'Alcoi 13: 53-127.

JUAN CABANILLES, J. (1994): "Estructuras de habitación en la Ereta del Pedregal (Navarrés, Valencia). Resultados de las campañas de 1980-1982 y 1990", Saguntum-PLAV 27: 67-97.

LEHMANN, J; DA SILVA, J; STEINER, C; NEHLS T; ZECH,W y GLASER, B. (2003): "Nutrient availability and leaching in an archaeological Anthrosol and a Ferralsol of the Central Amazon basin: fertilizer, manure and charcoal amendments", Plant and Soil 249: 343-357

MATA, C.; QUIXAL, D. y MORENO, A. (en prensa): "Estrategias de ocupación y explotación del entorno periurbano de Kelin", en Col-loqui Internacional El paisatge periurbà durant la Protohistòria i l'Antiguitat a la Mediterrània Occidental.

MIN, H. y ZHENG, SQ. (1993): "Time-space optimal convex Hull algorithms", en Symposium on Applied Computing (SAC): 687-693.

MOREIRA A. y SANTOS M. (2007): “Concave hull: A k-nearest neighbours approach for the computation of the region occupied by a set of points", en J. Braz, P.P. Vázquez y J. Madeiras (eds.), Proceedings of the Second International Conference on Computer Graphics Theory and Applications (Barcelona 2007). Institute for Systems and Technologies of Information: 61-68.

NICOSIA, C.; BALISTA, C.; CUPITÒ, M.; ERTANI, A.; LEORNARDI, G.; NARDI, S. y VIDALE, M. (en prensa): "Anthropogenic deposits from the Bronze Age site of Fondo Paviani (Verona, Italy): pedochemical and micropedological characteristics", International Quaternary. doi:10.1016/j.quaint.2010.10.006

NOCETE, F.; ORIHUELA, A..; ESCALERA, P.; LINARES, J. A.; OTERO, R. y ROMERO, J. C. (1995): "Prospecciones arqueológicas de superficie 
en el marco del Proyecto Odiel en 1992. I: Muestreo Valverde del Camino II. Huelva", Anuario de Arqueología Andaluza 1992. Vol. II. Actividades Sistemáticas: 199-207.

PASCUAL BENITO, J.LL. (2003): “Destrucció i recuperación del patrimonio. Intervencions arqueològiques en les sitges d'una aldea neolítica", en El patrimoni històric $i$ artístic de Cocentaina. La seua recuperació: 345-394.

PEÑA, J.L.; ENRIQUE, M.; GRAU, E. y MARTÍ, MaA. (1996): El poblado de la mola d'Agres: homenaje a Milagros Gil-Mascarell Boscà, Memòries d'excavacions arqueolòiques 1 . Generalitat Valenciana: Conselleria de Cultura, Ciència i Educació. València.
PIQUERAS HABA, J. (1995): Geografia de les comarques valencianes. Ed. Foro.

PORTA CASANELLAS, J. (2003): Edafología para la agricultura y el medio ambiente. Ed. Mundi-Prensa.

RENFREW, C. y BANH, P. (1993): Arqueología. Teorías, Métodos y Prácticas. Akal.

STEVANOVIC, M. (1997): "The age of clay: The social dynamics of house destruction", Journal of Anthropological Archaeology 16 (4): 334-395.

WILSON, C.A.; DAVISON, D.A. y CRESSER, M.S. (2009): "An evaluation of the sites specificity of soil elemental signatures for identifying and interpreting former functional areas", Journal of Archaeological Science 36 (10): 2327-2334. doi:10.1016/j. jas.2009.06.022

FECha DE ENTRADA: 19-10-2010

FeCHA DE ACEPTACIÓN: 18-01-2011 